\title{
GENERATION OF TRANSLUCENT LANGUAGE BY SUPERIMPOSITION OPERATION
}

\author{
C. Annal Deva Priya Darshini ${ }^{1}$ and V. Rajkumar Dare ${ }^{2}$ \\ Department of Mathematics, Madras Christian College, India
}

\begin{abstract}
We have introduced a new operation called the superimposition operation. The translucent language generated by a given superimposition operation and a language $L$ is the set of words generated by the superimposition of any two words in L. In this paper we study the properties of translucent languages. We also introduce a variant of the operation called Superimposition under control. We examine the properties of languages under this operation.
\end{abstract}

\section{Keywords:}

Translucent Words, Translucent Languages, Superimposition Under Control

\section{INTRODUCTION}

Research on Operation on words is a vast and independent area of study under combinatorics on words. The catenation operation is studied widely in Formal Language Theory in connection with composition of languages and their description through grammars. Several generalizations of the catenation operation like shuffle operation, insertion and deletion operations, shuffle on trajectories were introduced and studied by many authors in $[6,8]$. The shuffle operation is useful in modelling parallel composition of words and languages. Theoretical generalization to the case of infinite words was studied in detail by Kadrie et al. in [1]. Another notable operation is the Collage operation on words [4]. Motivated by different real life examples, we introduced a new operation called the superimposition operation in [2]. In this paper we study the properties of languages in relation to the superimposition operation. We take our inspiration from three different real life scenarios to introduce this operation. The scope of this study is to model picture composition in Astronomy and explore the connections between Formal Languages and Astronomy.

In Astronomy, information from celestial objects is collected through special colour filters on telescopes. A colour picture is composed by overlapping multiple layers of colours using advanced image processing software. The output or final colour that we perceive is the result of the superimposition of many layers.

We model this phenomenon as an operation on words and languages. The proposed model considers each letter of the alphabet as a translucent unit like a filter so that when one is placed on top of the other, we would be able to see through. The result is a different colour or word. The rules of superimposition specify what the resulting word would be. We can define an entire class of superimposition operations on a given alphabet.

This paper is organized as follows: Section 2 gives the preliminaries required for the study. In section 3 we introduce the concept of translucent language associated with any language and study the properties. Iterated superimposition of a language is obtained by iterations of the same operation. We study the properties of iterated superimpositions also in this section. In section 4 we introduce a variation in the operation by imposing a control. We study control languages and superimposition under control in this section. The conclusion gives the relevance and future work.

\section{BASIC DEFINITIONS}

Let $\Sigma$ be a finite alphabet and $\Sigma^{*}$ be the collection of all words over $\Sigma$ including the empty word $\lambda$. Let $\Sigma^{+}=\Sigma^{*}-\{\lambda\}$. For $w \in \Sigma^{+}$, $\operatorname{alph}(w)$ is the elements of $\Sigma$ in $w$. The length of a word $w$ is $|w|$. A word is denoted by $w=w_{1} w_{2} \ldots w_{n}$ where each $w_{i}$ is in $\Sigma$. A word $u$ is a factor of $w$ if there exists $x$ and $y$ such that $w=x u y$. The word is a prefix or suffix of wif $w=u y, w=x u$ respectively. If $w$ $=w_{1} w_{2} \ldots w_{n}$ where each $w_{i}$ is in $\Sigma$ then $w^{R}=w_{n} w_{n-1} \ldots w_{l}$. A language $L$ is a set of words or a subset of $\Sigma^{*}$. $L^{c}$ denotes the complement of a language. by,

The shuffle operation [8], denoted by $\diamond$, is defined recursively

$$
(a u \diamond b v)=a(u \diamond b v) \cup b(a u \diamond v), \text { and }(u \diamond \lambda)=(\lambda \diamond u)=\{u\}
$$

where, $u, v \in \Sigma^{*}$ and $a, b \in \Sigma$.

The shuffle of two languages $L_{1}$ and $L_{2}$ is $L_{1} \diamond L_{2}=\bigcup_{u \in L_{1}, v \in L_{2}} u \diamond v$.

Example: $(a b \diamond b c)=\{a b b c, a b c b, b a b c, b a c b, b c a b\}$.

The Collage operation on words is defined in [4] as: Given a subset $W \subseteq \Sigma^{*}$ of patches, the operation of Collage consists of producing words in $(\Sigma \cup\{\Delta\})^{*}$, ( $\Delta$ is a new symbol not in $\left.\Sigma\right)$, by starting with a word of the form $\Delta^{m}$ and then repeatedly replacing random factors of the current word with elements of $W$. A word thus obtained is called a Collage of $W$. Define $C^{0}(W)=\Delta^{*}$ and for all $k \geq 0, C^{k+1}(W)=\left\{w[i \leftarrow z] \mid w \in C^{k}(W)\right.$

$$
z \in W, 1 \leq i \leq|w|-|z|+1
$$

Example: Consider $n=11$ and assume the words $a b a, b b b b c$, $c a$, abaabcab belong to the subset $W$ and are placed respectively at the positions 2, 4, 10 and 1 in that order. The resulting word is at the top of the following Table.1.

Table.1. Collage of $W$

\begin{tabular}{ccccccccccc}
$a$ & $b$ & $a$ & $a$ & $b$ & $c$ & $a$ & $b$ & - & $c$ & $a$ \\
\hline$a$ & $b$ & $a$ & $a$ & $b$ & $c$ & $a$ & $b$ & & & \\
& & & & & & & & & $c$ & $a$ \\
& & & $b$ & $b$ & $b$ & $b$ & $c$ & & & \\
& $a$ & $b$ & $a$ & & & & & & & \\
\hline 1 & 2 & 3 & 4 & 5 & 6 & 7 & 8 & 9 & 10 & 11
\end{tabular}


Position 4 is covered by the occurrences $a b a, b b b b c$ and $a b a a b c a b$. Position 9 is covered by no occurrence and position 2 is covered by the occurrences of $a b a$ and $a b a a b c a b$.

\section{TRANSLUCENT LANGUAGES}

We introduce the concept of superimposition operation and translucent languages generated by a language. This language has some interesting properties.

We recall the definition of superimposition operation introduced in [2].

For $a, b \in \Sigma, a \otimes b=c$ where $c$ is also in $\Sigma$ is called the superimposition operation. This operation can be extended to $\Sigma^{*}$.

\section{Definition 3.1}

Let $u, v \in \Sigma^{+}$and $u=u_{1} u_{2} \ldots u_{n}, v=v_{1} v_{2} \ldots v_{m}$ where $u_{i}, v_{j} \in \Sigma$, for $i=1,2, \ldots, n, j=1,2, \ldots, m$.

a) If $|u|=|v|$, then $u \otimes v=\left(u_{1} \otimes v_{1}\right)\left(u_{2} \otimes v_{2}\right) \ldots\left(u_{n} \otimes v_{n}\right)$

b) If $|u|>|v|$, and if $u=u^{\prime} u^{\prime \prime}$ where $\left|u^{\prime}\right|=|v|=m$, then $u \otimes v=\left(u_{1} \otimes v_{1}\right)\left(u_{2} \otimes v_{2}\right) \ldots\left(u_{m} \otimes v_{m}\right) . u^{\prime \prime}$.

c) If $|u|<|v|$, and if $v=v^{\prime} v^{\prime \prime}$ where $\left|v^{\prime}\right|=|u|=n$, then $u \otimes v=\left(u_{1} \otimes v_{1}\right)\left(u_{2} \otimes v_{2}\right) \ldots\left(u_{n} \otimes v_{n}\right) . v^{\prime \prime}$

d) For $u \in \Sigma^{*}, u \otimes \lambda=\lambda \otimes u=u$.

Given an alphabet, it is possible to define a class of superimposition operations on the alphabet. If $X=\{a, b\}$, it is possible to specify 16 different rules. The word $z$ generated from two words $u$ and $v$ is unique and is called a translucent word obtained from $u$ and $v$. We use a representation of the operation in the form of a table.

\section{Example 3.1}

Let $\Sigma=\{b, w, g\}$ and the operation $\otimes$ given by the following Table.2.

Table.2. Superimposition rule

\begin{tabular}{|c|c|c|c|}
\hline$\otimes$ & $b$ & $w$ & $g$ \\
\hline$b$ & $b$ & $b$ & $b$ \\
\hline$w$ & $b$ & $w$ & $g$ \\
\hline$g$ & $b$ & $g$ & $g$ \\
\hline
\end{tabular}

Let $x=(b w)^{5}, y=(w b)^{5}$ be words in $\Sigma^{*}$ then $x \otimes y=b^{10}$.

\section{Definition 3.2}

Let $a, b, c \in \Sigma$. The operation $\otimes$ is

a) associative if $a \otimes(b \otimes c)=(a \otimes b) \otimes c$,

b) commutative if $b \otimes a=a \otimes b$,

c) Idempotent if $a \otimes a=a$.

Some simple results of the operation are immediate.

Let $x, y, z$ be words in $\Sigma^{+}$. Then

a) $|x \otimes y|=\max \{|x|,|y|\}$,

b) $x \otimes y=y \otimes x$ if and only if $\otimes$ is commutative,

c) $x \otimes(y \otimes z)=(x \otimes y) \otimes z$ if and only if $\otimes$ is associative

The operation can be extended to languages.

\section{Definition 3.3}

Let $L_{1}, L_{2} \subseteq \Sigma^{+}$then the superimposition of $L_{1}$ and $L_{2}$ is $L_{1} \otimes L_{2}=\bigcup_{u \in L_{1}, v \in L_{2}} u \otimes v$. For a given language $L$,

$$
L_{\otimes}^{0}=\lambda, L_{\otimes}^{1}=L, L_{\otimes}^{i+1}=L_{\otimes}^{i} \otimes L .
$$

Then the star closure of a language is $L_{\otimes}^{*}=L_{\otimes}^{0} \cup L_{\otimes}^{1} \cup L_{\otimes}^{2} \ldots .$. and the positive closure is $L_{\otimes}^{+}=L_{\otimes}^{1} \cup L_{\otimes}^{2} \ldots$. for a given superimposition operation. The superimposition of two languages is always a non-empty language.

\section{Definition 3.4}

For a given language $L$, the translucent language generated by $L$ under the superimposition operation $\otimes$ is given by $T(L, \otimes)=\left\{w \in \Sigma^{+} / w=u \otimes v, u, v \in L\right\}$.

\section{Example 3.2}

Let $\Sigma=\{a, b\}$ and let the operation $\otimes$ be specified by Table.3.

Table.3. Superimposition rule

\begin{tabular}{|l|l|l|}
\hline$\otimes$ & $a$ & $b$ \\
\hline$a$ & $a$ & $b$ \\
\hline$b$ & $b$ & $b$ \\
\hline
\end{tabular}

Let $L$ be the set of words having exactly one $a$. The translucent language generated is $T(L, \otimes)=L \cup\left\{b^{n} / n>0\right\}$.

The translucent language generated by a language is either itself or different from the language. However, there are some special cases where the translucent language generated is identical to the language from which it is generated. We give an example where the translucent language is the same as the language from which it is generated.

\section{Example 3.3}

For the unary alphabet $\Sigma=\{a\}$, if $L=\left\{a^{n} / n>0\right\}$ then $T(L, \otimes)=L$

We give an illustration of the operation as a simulation on how a colour image is produced.

Generation of a colour picture involves the use of two main colour spaces - RGB and CMYK. The RGB colour model related to the way we perceive colour with the $r, g, b$ receptors in our retina. RGB uses additive colour mixing and is the basic colour model used in television or any other medium that projects colour with light. The secondary colours of RGB - cyan, magenta and yellow - are formed by mixing two of the primary colours and excluding the third colour. The four colour CMYK model used in printing lays down overlapping layers of transparent inks to form a colour picture. In the following example we generate a transclucent language by the superimposition of colours.

\section{Example 3.4}

Let $\Sigma=\{r, g, b, c, y, m, k\}$ denote the colours

$r-$ red

$g$ - green

$b$-blue

$c$-cyan 


$$
\begin{aligned}
& y \text {-yellow } \\
& m \text { - magenta } \\
& k \text { - black }
\end{aligned}
$$

and the superimposition operation be given by Table.4.

Let $L=\left\{(y m y)^{p} c^{2 q}, p, q>0\right\} \cup\left\{(m y m)^{p}\left(y^{q} m^{q}\right), p, q>0\right\}$ then $\left.T(L, \otimes)=L \cup\left\{r^{p}(g b)^{q}\right), p, q>0\right\}$.

We note that the superimposition operation on the colours is a commutative and associative operation.

Table.4. Superimposition rule on colours

\begin{tabular}{|c|c|c|c|c|c|c|c|}
\hline$\otimes$ & $r$ & $g$ & $b$ & $c$ & $y$ & $m$ & $k$ \\
\hline$r$ & $r$ & $k$ & $k$ & $k$ & $r$ & $r$ & $k$ \\
\hline$g$ & $k$ & $b$ & $k$ & $g$ & $g$ & $k$ & $k$ \\
\hline$b$ & $k$ & $k$ & $b$ & $b$ & $k$ & $b$ & $k$ \\
\hline$c$ & $k$ & $g$ & $b$ & $c$ & $g$ & $b$ & $k$ \\
\hline$y$ & $r$ & $g$ & $k$ & $g$ & $y$ & $r$ & $k$ \\
\hline$m$ & $r$ & $k$ & $b$ & $b$ & $r$ & $m$ & $k$ \\
\hline$k$ & $k$ & $k$ & $k$ & $k$ & $k$ & $k$ & $k$ \\
\hline
\end{tabular}

A black and white picture would look something like this.

\section{Example 3.5}

Let, $\Sigma$ be the alphabet $\{\square \square \square\}$

where, $\square \square$ denote the colours black, white and gray respectively and the superimposition operation be given by Table.5.

Let $L$ be the language, $\left\{(\square \square \square \square \square \square \square)^{n}, n>1\right\}$ then $T(L, \otimes)=L$.

Table.5. Superimposition rule on black and white colours

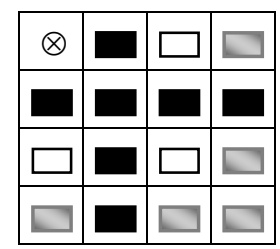

\section{Proposition 3.1}

If $L \subseteq \Sigma^{n}$ is closed with respect to a superimposition $\otimes$ then $T(L, \otimes)$ is also closed with respect to the superimposition operation.

Proof: Let $w_{1}, w_{2} \in T(L, \otimes)$. Since $w_{1} \in T(L, \otimes)$, we have $w_{1}=$ $x_{1} \otimes y_{1}$ for some $x_{1}, y_{1} \in L$. Similarly, $w_{2}=x_{2} \otimes y_{2}$ for some $x_{2}, y_{2}$ $\in \mathrm{L}$. Since $L$ is closed, $x_{1} \otimes y_{1}=x \in L$ and $x_{2} \otimes y_{2}=y \in L$. Now, $x \otimes y=z \in T(L, \otimes)$ by the definition of translucent languages. Hence $T(L, \otimes)$ is closed.

A language $L$ is commutative [6] if for any $w \in L$ contains all the words obtained from $w$ by arbitrarily permuting its letters. For a word $u=a_{1} a_{2} \ldots a_{k} \in \Sigma^{*}, k \geq 0$, we define $\operatorname{com}(u)=$ $\left\{a_{s(1)} a_{s(2)} \ldots a_{s(k)} \mid s\right.$ a permutation of $\left.\{1, \ldots, k\}\right\}$ that is, $\operatorname{com}(u)$ contains all the words obtained by arbitrarily permuting the letters of $u$. If $L \subseteq \Sigma *$ then $\operatorname{com}(L)=\bigcup_{u \in L} \operatorname{com}(u) . L$ is commutative if and only if $L=\operatorname{com}(L)$.

\section{Proposition 3.2}

If $L \subseteq \Sigma^{+}$is a commutative language then $T(L, \otimes)$ is also a commutative language.

Proof: Let $w \in T(L, \otimes)$. Then $w=x \otimes y$ for some $x, y \in L$. Since $L$ is commutative, $\operatorname{com}(x), \operatorname{com}(y) \in L$. This implies $\operatorname{com}(x)$ $\otimes \operatorname{com}(y) \in \mathrm{T}(L, \otimes)$ for all possible permutations of $\{1,2, \ldots, n\}$. Further, $\operatorname{com}(x) \otimes \operatorname{com}(y)=\operatorname{com}(x \otimes y)$ for each permutation.

\section{Proposition 3.3}

The translucent language $T\left(\Sigma^{n}, \otimes\right)$ is always a commutative language.

Proof: By definition, $\Sigma^{n}$ is commutative and $T\left(\Sigma^{n}, \otimes\right)=\Sigma^{n}$.

Note that $T(L, \otimes)=L_{\otimes}^{2}$.

\section{Definition 3.5}

The iterated superimposition of two languages is given by $L_{1} \otimes * L_{2}=\bigcup_{n=0}^{\infty}\left(L_{1} \otimes^{n} L_{2}\right)$, where

$$
L_{1} \otimes^{0} L_{2}=L_{1} \text { and } L_{1} \otimes^{n+1} L_{2}=\left(L_{1} \otimes^{n} L_{2}\right) \otimes L_{2} .
$$

We say that a superimposition operation is neutral if

a) The superimposition operation is given by the following rules: $a \otimes a=a \otimes b=b \otimes a=a, b \otimes b=b$,

b) $a \otimes a=a, a \otimes b=b, b \otimes a=a, b \otimes b=b$.

The neutral superimposition leaves the word unaltered or hides the word completely. We call the superimposition operation given by rule (i) as Type I neutral operation and the rule (ii) as Type II neutral operation.

\section{Proposition 3.4}

If $\otimes$ is a neutral operation of Type I or Type II then $T(L, \otimes)=$ $L$ for any $L \subseteq \Sigma^{+}$.

\section{Proposition 3.5}

If $\otimes$ is a neutral operation then we have

a) $L_{1} \otimes^{n} L_{2}=L_{1}$ for any $L \subseteq \Sigma^{+}$if the operation is of Type I and

b) $L_{1} \otimes^{n} L_{2}=L_{2}$ for any $L \subseteq \Sigma^{+}$if the operation is of Type II.

\section{Proposition 3.6}

If $\otimes$ is a neutral operation then we have

a) $T\left(L_{1} \otimes^{n} L_{2}, \otimes\right)=L_{1}$ for any $L \subseteq \Sigma^{+}$if the operation is of Type I and

b) $T\left(L_{1} \otimes^{n} L_{2}, \otimes\right)=L_{2}$ for any $L \subseteq \Sigma^{+}$if the operation is of Type II.

Proof follows from propositions 3.4 and 3.5.

\section{Proposition 3.7}

If $\otimes$ is idempotent then we have,

a) $L \subset T(L, \otimes)$ for any $L \subseteq \Sigma^{+}$,

b) $L \otimes^{n} L=L$ for any $L \subseteq \Sigma^{+}$.

Proof: If $x \in L$ then $x \otimes x=x$ since the operation $\otimes$ is idempotent. This implies $x \in \underline{T}(L, \otimes)$. 


\section{SUPERIMPOSITION UNDER CONTROL}

In this section, we introduce a variant of this operation where we restrict the superimposition of two words using a control language. Here, we subdivide both the words into factors using the control language as a guide and then allow the factors to superimpose.

We introduce a new alphabet $V=\{f, s\}$ to define the operation. Let $c \in V^{*}$ be the control word and $C \subseteq V^{*}$ be a control language.

\section{Definition 4.1}

Let $x=x_{1}, x_{2}, \ldots x_{m} \in \Sigma^{*}$ and $y=y_{1}, y_{2}, \ldots y_{n} \in \Sigma^{*}$ for $x_{i}, y_{i} \in \Sigma$, $i=1,2, \ldots m, j=1,2, \ldots n$.

Let $c=z_{1}^{n_{1}} z_{2}^{n_{2}} \ldots z_{k}^{n_{k}} \in V^{*}, z_{i} \in V, n_{i} \in \mathrm{N}$.

If $\quad|c|_{f}=|x|,|c|_{s}=|y|,|c|=|x|+|y|$, we define the superimposition of $x$ and $y$ controlled by $c \in V^{*}$ as

$$
x \otimes_{c} y=\left\{\begin{array}{l}
\left(x_{1} x_{2} \ldots x_{n_{1}}\right) \otimes\left(y_{1} y_{2} \ldots y_{n_{2}}\right) \ldots . ., z_{1}=f \\
\left(y_{1} y_{2} \ldots y_{n_{1}}\right) \otimes\left(x_{1} x_{2} \ldots x_{n 2_{1}}\right) \ldots ., z_{1}=s \\
\varphi, \text { otherwise }
\end{array}\right.
$$

Each $z_{i} \in V$ is repeated a certain number of times in c. The number of repetitions is counted by the indices $n_{1}, n_{2}, \ldots$ If $z_{1}$ is $f$ then $z_{2}$ is $s$. In such a case, the first $n_{1}$ letters of the first word is superimposed on the first $n_{2}$ letters of the second word. The $z_{i}$ alternate between $f$ and $s$.

Hence it is enough to check the first letter of $\mathrm{C}$. The superimposition is repeated until all the letters of the control word are exhausted. The word generated is unique and is of length $\max \left(n_{1}, n_{2}\right)+\max \left(n_{3}, n_{4}\right)+\ldots \max \left(n_{k-1}, n_{k}\right)$.

\section{Example 4.1}

Let $x=a_{1} a_{2} \ldots a_{8} \in \Sigma^{*}$ and $x=b_{1} b_{2} \ldots b_{5} \in \Sigma^{*}, c=f^{3} s^{2} f^{3} s f s f s$.

Note that $|c|_{f}=|x|,|c|_{s}=|y|,|c|=|x|+|y|$. The word $x$ is factorised as $x=u_{1} u_{2} u_{3} u_{4}$ and $y=v_{1} v_{2} v_{3} v_{4}$ using $c$ as a control. We have,

Therefore,

$$
\begin{gathered}
u_{1}=a_{1} a_{2} a_{3}, u_{2}=a_{4} a_{5} a_{6}, u_{3}=a_{7}, u_{4}=a_{8}, \\
v_{i}=b_{1} b_{2}, v_{2}=b_{3}, v_{3}=b_{4}, v_{4}=b_{5} .
\end{gathered}
$$

$$
x \otimes_{c} y=\left(a_{1} a_{2} a_{3} \otimes b_{1} b_{2}\right)\left(a_{4} a_{5} a_{6} \otimes b_{3}\right)\left(a_{7} \otimes b_{4}\right)\left(a_{8} \otimes b_{5}\right)
$$

\section{Definition 4.2}

If $c$ is a control language over $V^{*}$ then $x \otimes_{C} y=\bigcup_{c \in C} x \otimes_{c} y$.

If $c$ is the empty set then $x \otimes_{c} y=\varphi$.

Also if $L_{1}, L_{2} \subseteq \Sigma *$ then $L_{1} \otimes_{C} L_{2}=\left\{x \otimes_{C} y / x \in L_{1}, y \in L_{2}\right\}$.

Intuitively, a control language gives us a guide to subdivide two words in a particular way and to superimpose the subwords. The factorization of $x$ and $y$ into subwords using a control word gives us an alternate definition of superimposition under control.

\section{Definition 4.3}

Let us denote the run of a letter in $c \in V^{*}$ be the number of consecutive occurrences of the letter in $c$.

For example, if $c=f^{3} s^{2}$ then $\operatorname{run}(f)=3$ and $\operatorname{run}(s)=2$.
If $c$ is a word with more than one non - consecutive occurrence of the letter $f$ or $s$ then we denote the runs as $\operatorname{run}_{i}(f)$.

If $c=f^{3} s^{2} f^{3} s f s f s$ then $\operatorname{run}_{1}(f)=3, \operatorname{run}_{2}(f)=3, \operatorname{run}_{3}(f)=1$, $\operatorname{run}_{4}(f)=1, \operatorname{run}_{1}(s)=2, \operatorname{run}_{2}(s)=\operatorname{run}_{3}(s)=\operatorname{run}_{4}(s)=1$.

Then if $\sum_{i} \operatorname{run}_{i}(f)=|x|$ and $\sum_{j} \operatorname{run}_{j}(s)=|y|$ we factorise $x=$ $u_{1} u_{2} \ldots, y=v_{1} v_{2} \ldots$ where $\left|u_{i}\right|=\operatorname{run}_{i}(f)$ and $\left|v_{j}\right|=\operatorname{run}_{j}(s)$ for $i, j=$ $1,2, \ldots$.

In such a case, if $t \in V$ denotes $f$ or $s$ and $c=t V^{*}$ we have

$$
x \otimes_{c} y=\left\{\begin{array}{l}
\left(u_{1} \otimes v_{1}\right)\left(u_{2} \otimes v_{2}\right) \ldots . ., t=f \\
\left(v_{1} \otimes u_{1}\right)\left(v_{2} \otimes u_{2}\right) \ldots . ., t=s \\
\varphi, \text { otherwise }
\end{array}\right.
$$

\section{Example 4.2}

Let $\Sigma=\{a, b\}$ and $V=\{f, s\}$. Let $c \in V^{14}$ and let $x, y \in \Sigma^{7}$ be words on $\Sigma$. Let $x=a b a a b a b, y=a b b b a a b$.

Then,

a) if $c=f^{7} s^{7}$ we have $x \otimes_{c} y=x \otimes y$

b) if $c=(f s)^{7}$ we have $x \otimes_{c} y=x \otimes y$

c) if $c=f^{7}$ we have $x \otimes_{c} y=x$

d) if $c=s^{7}$ we have $x \otimes_{c} y=y$

e) if $c=s^{7} f^{7}$ we have $x \otimes_{c} y=y \otimes x$.

\section{Definition 4.4}

Let $C$ be a control set. We say that $C$ is commutative if and only if the operation $\otimes_{c}$ is commutative, that is, $x \otimes_{c} y=y \otimes_{c} x$ for all $x, y \in \Sigma^{*}$.

Let $\aleph$ be the family of all commutative sets of control words.

\section{Proposition 4.1}

If $\left(C_{i}\right)_{i \in I}$ is a family of control languages such that $\left(C_{i}\right)$ is a commutative control language for all $i \in I$ then their intersection $C^{\prime}=\bigcap_{i \in I} C_{i}$ is also a commutative control language.

Proof:

Let $u, v \in \Sigma^{*}$ and $w \in u \otimes_{C^{\prime}} v$. Then it follows that $w \in u \otimes_{C_{i}} v$ for all $i \in I$. But, each $\left(C_{i}\right)$ is commutative and hence $w \in v \otimes_{C_{i}} u$ for all $i \in I$. Therefore, $w \in v \otimes_{C^{\prime}} u$. Thus, we have $u \otimes_{C^{\prime}} v=v \otimes_{C^{\prime}} u$ which implies that $C^{\prime}=\bigcap_{i \in I} C_{i}$ is also a commutative control language.

\section{Definition 4.5}

Let $C$ be a control language. The commutative closure of $C$ denoted by $\bar{C}$ is given by $\bar{C}=\bigcap_{C \subseteq C^{\prime}, C^{\prime} \in N^{\prime}} C^{\prime}$.

Corollary: For all $C \subseteq\{f, s\}^{*}, \bar{C}$ is a commutative control language.

Remark: $\bar{C}$ is the smallest control language that contains $C$.

\section{Definition 4.6}

A control language $C$ is associative if and only if $\otimes_{c}$ is associative. We have $x \otimes_{c}\left(y \otimes_{c} z\right)=\left(x \otimes_{c} y\right) \otimes_{c} z$ for all $x, y, z \in \Sigma^{*}$. 


\section{Proposition 4.2}

If $\left(C_{i}\right)_{i \in I}$ is a family of control languages such that $\left(C_{i}\right)$ is an associative control language for all $i \in I$ then their intersection $C^{\prime \prime}=\bigcap_{i \in I} C_{i}$ is also an associative control language.

Proof: Let $x, y, z \in \Sigma^{*}$ and let $w \in x \otimes_{C^{\prime \prime}}\left(y \otimes_{C^{\prime \prime}} z\right)$. Then it follows that $w \in x \otimes_{C_{i}}\left(y \otimes_{C_{i}} z\right)$ for all $i \in I$. But, each $\left(C_{i}\right)$ is associative and hence $w \in\left(x \otimes_{C_{i}} y\right) \otimes_{C_{i}} z$ for all $i \in I$. Therefore, $w \in\left(x \otimes_{C^{\prime \prime}} y\right) \otimes_{C^{\prime \prime}} z$. Thus, we have $x \otimes_{C^{\prime \prime}}\left(y \otimes_{C^{\prime \prime}} z\right)$ $=\left(x \otimes_{C^{\prime \prime}} y\right) \otimes_{C^{\prime \prime}} z$ which implies that $C^{\prime \prime}=\left.\right|_{i \in I} C_{i}$ is also an associative control language.

\section{Definition 4.7}

Let $C$ be a control language. The associative closure of $C$ denoted by $\overline{\bar{C}}$ is $\overline{\bar{C}}=\bigcap_{c \subseteq C^{\prime \prime}, C^{\prime} \in \mathrm{A}} C^{\prime \prime}$ where $A$ is the family of all associative control languages.

\section{Proposition 4.3}

The associative closure $\overline{\bar{C}}$ is also an associative control language.

\section{Proposition 4.4}

Let $L_{1}, L_{2} \subseteq \Sigma^{*}$ be regular languages. Then $L_{1} \otimes L_{2}$ is regular.

Proof: We give the automata to prove that the superimposition of two regular languages is also regular. Let $A_{1}$ and $A_{2}$ be two finite automata accepting the languages $L\left(A_{1}\right)$ and $L\left(A_{2}\right)$. Then we can find an automaton $A$ such that $L(A)=L\left(A_{1}\right) \otimes L\left(A_{2}\right)$. Let $A_{i}=\left(Q_{i}, \Sigma, \delta_{i}, q_{0}^{i}, F\right)$ for $i=1,2$ be the two finite automata accepting languages $L\left(A_{1}\right)$ and $L\left(A_{2}\right)$. We construct the automaton $A=\left(Q, \Sigma, \delta, q_{0}, F\right)$ as $Q=Q_{1} \times Q_{2}, F=F_{1} \times F_{2}, q_{0}=\left(q_{0}^{1}, q_{0}^{2}\right)$ and $\delta\left(\left(q_{i}^{1}, q_{j}^{2}\right),(a, b)\right)=\left(\left(q_{k}, q_{l}\right), c\right)$ where, $\delta_{1}\left(q_{i}^{1}, a\right)=q_{k}, \delta_{2}\left(q_{j}^{2}, b\right)=$ $q_{l}$ and $a \otimes b=c$ is the rule of superimposition specified.

The automaton $A$ accepts an input $w$ if and only if $A_{l}$ accepts $w_{1}$ and $A_{2}$ accepts $w_{2}$ where the transition rules are $\delta\left(\left(q_{i}^{1}, q_{j}^{2}\right),\left(w_{1}, w_{2}\right)\right)=\left(\left(q_{k}, q_{l}\right), w\right),\left(q_{k}, q_{l}\right) \in F$ for $\delta_{1}\left(q_{i}^{1}, w_{1}\right)=q_{k}$, $q_{k} \in F_{1}$ and $\delta_{2}\left(q_{j}^{2}, w_{2}\right)=q_{l}, q_{l} \in F_{2}$ and $w_{1} \otimes w_{2}=w$.Then $L(A)=$ $L\left(A_{1}\right) \otimes L\left(A_{2}\right)$.

\section{Proposition 4.5}

For every language, $L \subseteq \Sigma^{*}, \Sigma=\{a, b\}$ there exists a control language $C$ such that $L=a * \otimes_{c} b^{*}$.

Proof: Let $\varphi$ be a morphism, $\varphi(a)=f, \varphi(b)=s$ and let $C=\varphi(L)$ then $L=a * \otimes_{c} b^{*}$.

\section{Theorem 4.1}

Let $V=\{f, s\}$. Let $C \subseteq V *$ be a control language, then the following are equivalent:

a) For all regular languages $L_{1}, L_{2} \subseteq \Sigma^{*}$, the language $L=L_{1} \otimes_{c} L_{2}$ is a regular language,

b) $C$ is a regular language.
Proof: To prove (i) implies (ii). Assume $L_{1}=f *$ and $L_{2}=s *$ then $L_{1} \otimes_{c} L_{2}=C$. Therefore, $C$ is a regular language.

To prove (ii) implies (i), Assume that $C$ is a regular language. Let $L_{1}, L_{2}$ be two regular languages over the same alphabet $\Sigma$. Let $A_{i}=\left(Q_{i}, \Sigma, \delta_{i}, q_{0}^{i}, F\right)$ for $i=1,2$, be finite automata such that $L\left(A_{i}\right)=L_{i}$ for $i=1,2$. Also, let $A_{c}=\left(Q_{c}, V, \delta_{c}, q^{c}, F_{c}\right)$ be an automaton such that $L\left(A_{c}\right)=C$. Define an automaton $A=\left(Q, \Sigma, \delta, Q_{o}, F\right)$ such that. $L(A)=L_{1} \otimes_{c} L_{2}$ The input is accepted by if and only if $A_{1}, A_{2}, A_{c}$ are in accepting states. Then $L_{1} \otimes_{c} L_{2}$ is a regular language. We have,

$$
Q=Q_{1} \otimes Q_{c} \otimes Q_{2} \text { and } Q_{o}=\left\{q_{o}^{1}, q_{o}^{2}, q_{o}^{c}\right]
$$

where, $F=F_{1} \otimes F_{c} \otimes F_{2}$ and $\delta\left(q_{1}^{1}, c, q_{2}^{2}, a, b\right)=\left(q_{1}, q_{2}, c\right)$, $\delta_{1}\left(q_{1}^{1}, f, a\right)=q_{1}, \delta_{2}\left(q_{2}^{2}, s, b\right)=q_{2}, a \otimes_{c} b=c, c \in V^{*}$.

Define the transformation $\sigma:\{f, s\} \rightarrow\{f, s\} *$ as $\sigma(f)=s, \sigma(s)=f$. Then we have the following property of superimposition under control.

\section{Proposition 4.6}

Let $L_{1}, L_{2} \subseteq \Sigma^{*}$ and $C \subseteq V^{*}$ be any two languages and a control language. Let $x_{1} \in L_{1}, x_{2} \in L_{2}$ and $c_{1}, c_{2} \in C$. We have $x_{1} \otimes_{c} x_{2}=x_{2} \otimes_{c} x_{1}$ if and only if $c_{2}=\sigma\left(c_{1}\right)$.

\section{Definition 4.8}

A control language $C$ is commutative if and only if $C=\sigma(C)$.

\section{Proposition 4.7}

If $C$ is a regular control language then it is decidable whether or not $C$ is commutative.

Proof: If $C$ is a regular language then $\sigma(C)$ is also regular. Hence the equality, $C=\sigma(C)$ is decidable.

\section{CONCLUSION}

We have introduced the translucent language associated with a language generated by the operation and studied the properties. The natural extension to arrays is an obvious direction in which the work can proceed. Expressions involving superimpositions were introduced in [2]. This study can be taken further.

\section{REFERENCES}

[1] Ahmad Kadrie, V. Rajkumar Dare, D.G. Thomas and K.G. Subramanian, "Algebraic Properties of the Shuffle Over $\omega$ Trajectories”, Information Processing Letters, Vol. 80, No. 3, pp. 139-144, 2001.

[2] C. Annal Deva Priya Darshini and V. Rajkumar Dare, "Superimposition Operations on Words", Proceedings of National Conference on Mathematics and Computer Applications, pp. 48-52, 2015.

[3] Christian Choffrut and Juhani Karhumaki, "Combinatorics of words in Handbook of Formal Languages", Vol. 1, Handbook of Formal Languages, Springer, pp. 329-438, 1997. 
[4] Christian Choffrut and Berke Durak, "Collage of TwoDimensional Words",Theoretical Computer Science, Vol. 340, No. 2, pp. 364-380, 2005.

[5] J.E. Hopcroft and J.D. Ullman, "Introduction to Automata Theory, Languages and Computation", Narosa Publishing House, 1979.

[6] Masami Ito, Lila Kari and Gabriel Thierrin, "Shuffle and Scattered Deletion Closure of Languages", Theoretical Computer Science, Vol. 245, No. 1, pp. 115-133, 2000.
[7] Jean Berstel and Dominique Perrin, "The Origins of Combinatorics of Words", European Journal of Combinatorics, Vol. 28, No. 3, pp. 996-1022, 2007.

[8] A. Mateescu, G. Rozenberg and A. Salomaa, "Shuffle on Trajectories: Syntactic Constraints", Theoretical Computer Science, Vol. 197, No. 1-2, pp. 1-56,1998.

[9] J.E. Tremblay and R. Manohar, "Discrete Mathematical Structures with Applications to Computer Science", Tata McGraw Hill, 1997. 